\title{
Index of Names, Subjects and Passages
}

Accius, ed. Dangel Philocteta: 40; fr. 2: 40

Adonis 152

Aelian $V H$ 12.32: 67, 89; fr. (Domingo-Forasté) 10h, $\mathrm{i}^{1}: 13$

Aelius Aristides, ed. Keil 3.50: 63, 22.2: 13 and 15, 22.7.1: 17, 22.12: 15, 22.13: 10, 49.48: 122, fr. vol. 2.469 § 5: 41

Aelius Dionysius, ed. Erbse к 30:77, $\mu$ 2: 13

Aeschines and his mother's Mysteries: 68; 51; 3.130: 5

Aeschylus Cho. 490: 62; fr. (Radt) 97: 40, 387: 13, 451s.70: 62

Akousilaos FGrH 2 F $20=$ F 20 Fowler: 35, 39

Alcibiades 10, 52

Alexander of Abonuteichos, Mysteries 9, 15, 155

Alexander Polyhistor FGrH 273 F 19ab: 204, F 79 (4): 204, F 93: 89

Alexis, ed. Kassel/Austin fr. 183: 29

Ambrosiaster 133-34; Quaestiones 114.11: 133

Ambrosius Myst. 1.2: 163

Anchises 198-99

Andania, Mysteries 86-96; admission: 8889; agônothetês: 91; books: 96; cista mystica: 91-92; clothes: 89; dances: 95-96; Demeter: 92-93; Eleusinian influence: $95-96$; fair: 96 ; fee: 89 ; gods: 90-91; Great Gods: 93; Hagna: 93; headgear: 94, 96; Hermes: 93; hierothytai: 91; initiands: 94-95; lustration: 94; Mnasistratos: 88, 95-96; mystagogues: 94; mystic drama: 96; piloi: 92-93; pipers: 91, 95; priests/esses: 88, 90-92, 95; procession: $89-90,94$; ram: 90 , 93; rhabdophoroi: 95; sacred maidens: 92, 95; sacred men/women: 92, 94, 96; sacrifices: 89-91, 93, 95; sanctuary: 86-87; tents: 94; thoinarmostria: 92; two degrees: 95-96; Urgeschichte: 87; wreath: 96 ; yearly: 88

Andocides Myst. 12: 4, 29: 3, 15

Androtion FGrH $324 \mathrm{~F} \mathrm{80:} 40$

Anonymus Londinensis, ed. Jones 37.34: 172
Anthologia Latina, ed. Riese 6.245: 54, 7.89.5: 183

Anthologia Palatina 4.1.47-8: 195, 7.25.6: 199, 7.485: 107, 7.748: 194, 9.688: 107, 16.30: 189

Antinoos, Mysteries 154

Antonius Diogenes 201

Apocalypse of Abraham 199

Apocalypse of James 159

Apocalyse of Paul 13: 199, 21: 197

Apocalypse of Peter 180; 15.16: 199

Apollo A. Carinus: 167; A. Karneios: 55, 90, 96; and laurel: 197; A. Pythaios 86

Apollodorus FGrH $244 \mathrm{~F} \mathrm{89:173,} \mathrm{F} \mathrm{110b:} \mathrm{11,}$ F 139: 72

Apollodorus 1.1.2: 194, 1.4.1: 189, 1.5.1: 174, 1.5.3: 191, 2.1: 194, 2.2.1: 194; 2.5.12: 191-92, 3.10.3: 72, 3.10.4: 194; Ep. 1.23f: 192

Apollonius Rhodius 1.536-41: 57, 761-62: 189, 915-18: 23, 920-21: 27; 2.238-40: 195, 561-73: 195; 1270: 195; 3.62: 189, 541-54: 195; 4.125-26: 195, 596ff: 197

Apuleius and Isis: 114-25; sex scene: 115; Apol. 55.8: 109; De deo Socratis 8: 122; Met. 3.15.1: 120; 6.2: 6, 11.2: 121, 2.28: 122, 3.5: 121, 3.15: 122, 4.30: 122, 5.1: 121-22, 6.6: 121, 6.22: 122, 10.1-2: 121, 13-15: 115, 14.3: 121, 18.3: 116, 19-20: 115, 21: 116, 21.7: 121, 22.3: 116, 22.8: 118, 23.1: 116, 23.4: 120, 23.5-7: 121, 24 : 124, 24.2: 121, 24.3: 124, 25: 122, 25.5: 116, 27.4: 121, 28.4-6: 116, 30.1-2: 116 Arcadia, Mysteries 80-85; yearly; 82 Archemachus FGrH 424 F 6: 62 Ares Thracian: 56 Argonauts and initiation: 57; and Jenseitsfahrt: 195; and Lemnos: 40; and Orpheus: 57; and Samothrace: 23 Aristophanes Eccl. 443: 174, 1069: 52; Lys. 558: 49; Nu. 135: 77, 143: 148, 258: 148; Pax 277-78: 29, 1161-65: 40; Plut. 409-415: 7; Ra. 137-42: 184, 147: 198, 149-50: 198, 159: 6 and 107, 161ff: 192, 
186: 199, 269-70: 184, 313: 6, 314: 9, 316-17: 6, 326: 197, 330: 16, 336: 5, 342-43: 7, 369: 4, 374-75: 7, 389-93: 7, 404-06: 17, 409-15: 7, 431ff: 192 and 198, 448-55: 197, 450-51: 8, 671: 62, 943: 68, 1032-34: 58 and 198; Thesm. 281: 173, 287: 62, 585: 173, 623: 173, 834: 172, 893: 173, 990: 71, 995: 71, 1045: 173; Ve. 8: 52, 119-20: 52-53 and 97, 141-44: 50, 1362-65: 7; fr. 384 (Kassel/Austin): 38, fr. 506: 68

Aristomenes 87-88

Aristophon, ed. Kassel/Austin fr. 12: 61

Arkandisziplin 147, 162

Arnobius 2.53: 183, 4.14: 32, 5.19: 103 and 109, 5.26: 3

Arretophoria 169

Arrian Epict. 3.21.16: 6 and 13, 3.21.16: 10

Artemidorus 2.37: 99, 4.39: 102

Asclepiades FGrH 697 F 9: 60

Askalaphos 191

Assman, J. 144

Asterius, ed. Datema 10.9.1: 11-12

astrology and Egyptian priests: 117

Athanasius Apol.1.11: 163

Athenaeus 1.21e: 6, 2.40d: 4, 4.148bc: 103, 5.197a: 190, 11.496ab: 17, 10.428f : 40, 13.590f: 5

Athenakon FGrH 546 F 1: 29

Attis 152, 161

Augustine Civ. 4.31: 156

Austin, R. 181, 184, 193-94, 197

Bacchanalia 100, 105, 120

Bacchic Mysteries 70, 101; bakchoi: 70, 72; in Cumae: 72-73, 79; in Ephesus: 70-71; lênai: 70; mystai: 70; reincarnation: 75

Bacchylides 5.76-175: 191, 11.77: 194

bakchos see Orphism

baptism 152, 157; and Mysteries: 157; as mystêrion/mysterium: 162

Barth, K. 144

Baronio, C. 146

Baubo 65, 174

Bible 1 Sam 4.1-11: 42; Esther 1.6: 190; Wisdom 2.22, 6.22: 149: Matth. 4.8: 199; Mark 4.11: 150; Rom 11.25: 150; I Cor 2.1,
4.1: 150; Philipp. 4.11-13: 150; Col. 2.18:

150; Rev. 21.10: 199

boukolos 6

Bourdieu, P. 160

boutês 66

Breasted, J. 145

Brimo: 73

Brown, D. 144

Buddhism 140, 142

Bunsen, C.C.J. 59

Burkert, W. XI, XIII, 1, 32, 46, 138, 153

Callimachus H. 2.2: 182, 3.60-61: 193; fr. 43.117 Harder: 62, fr. 63.9-12 H/Pf: 172, fr. 113e H. = 115 Pfeiffer: 42; fr. 199 Pf = Dieg. VIII.37-39; fr. 643: 62, fr. 723: 36, fr. $804: 74$

Car/ia 166-68

Casaubon, I. IX, 145-47

Catullus 64.260: 77, 182

Cecrops 167

Censorinus DND 18.2: 102

Cerberus 186

Cercops 60

Chaeremon fr. (Van der Horst) 10: 117 and 125, fr. 20D: 118

Chamoux, F. 168-69

Champollion, J.-F. 145

Charon 184-85

Chrysippus SVFII 316.12: 58

Cicero Leg. 2.36: 19; ND 1.41: 58, 1.119: 9 and 40, 3.56: 32, 3.89: 29; Rep. 6.11: 199; Verr. 4.99: 172

Cinesias 97

cistophoric coins 108-09

Cleanthes SVF1. no. 538: 10

Clement of Alexandria Paed. 1.26.1-2; Protr. 2.12: 10, 2.13.5-14.1: 160, 2.15.3: 151, 2.16.1: 63, 2.17.1: 168, 2.18.1: 109, 2.21.2: 3, 2.22: 108, 12.120.1: 151, 160; Strom. 1.21.131.3: 60, 5.11: 3, 5.4.19.3: 118

Cleomedes, ed. Todd Caelestia, 2.1.498-9: 174

Clinias 49, 51

Commodianus Instruct. 1.13: 131

Conon FGrH 26 F 1.21: 33, F1.45: 57 
Constantine 162

Constitutiones Apostolicae 3.16.1: 70; 8.11.11: 162

Cornelius Labeo fr. (Mastandrea) 7: 187

Crinagoras Anth. Pal. 11.42: 19

Critias Pirithous: 192; TrGF 43 F 2: 17

Cumae Bacchic Mysteries: 72-73, 79

Cumont, F. X-XI, 110, 141

Curtius Rufus 4.13.12: 126

Cybele 36, 38, 39

Cybele and Attis, Mysteries 161

Cyclopes 193-94

Cyril of Jerusalem Cat. myst. 1.1, 4.1, 5.4.9: $163,5.20,23: 162,6.29: 163$

Daillé, J. 147

Damastes, ed. Fowler F 11b: 58

Damophon 84

Danae 188

Dardanos 36

Deioi 65

Demeter D. Achaia: 168; D. Amphiktionis: 168; and Anaklêthra: 176; in Andania: 87; and Car: 166-68; D. Chthonia: 75; and civilization: $167,169,171$; and Dionysos: 75 ; in Eleusis: $8,10,11,15$; hymn to: 77; D. Malophoros: 177-79; in Megara: 166-79; Mysteries: 151; and political power: 167-68, 172; temples: 171-72; D. Thesmios: 171 ; D. Thesmophoros: 167-68, 170-71

Demetrios fr. (Gaede) 61= FGrH $2013 \mathrm{~F}$ 61: 32, fr. $62=F G r H 2013$ F 62: 33

Demetrios of Kallatis FGrH 86 F 6:172

Democritus, ed. Diels/Kranz B 230: 1

Demosthenes In Neaeram 21; 18.259: 51 and 68, 19.199: 68, 21.158: 7, 54.8: 62, 59.21: 4

Derveni Papyrus 55, 59, 61, 79; dialect mixture: 64; VII.7-11: 63 and 182, XVII.12: 62, XX: 69; XXII.11-12: 65, XXIII.4: 62, $\mathrm{XXV} .14: 62$

dervishes 52

Dido 187

Dieterich, A. 180

Detienne, M. 175

Diasia 4-5
Dio Cassius 51.4.1: 196

Dio Chrysostom 4.90: 98, 12.27: 3, 12.33: 51

Diodoros Pasparos 41

Diodorus Siculus 1.22.7: 108, 1.96.5: 197, 3.55.9: 27 and 31, 3.63.3-4: 123, 4.2.2-3: 123, 4.26.1: 192, 4.63.4: 192, 4.68.2: 189, 5.4.6: 174, 5.47.14-16: 25, 5.48.4: 27 and 182, 5.48.5, 5.49.1: 28 , 5.49.2: 36, 5.49.5: 27, 5.49.6: 23, 33; 6.47-49: 33, 6 fr. 7: 189, 10.9.6: 89

Diogenes Laertius 2.130: 91; 6.59: 29

Diogenianus 6.98: 6

Dioklos/es 177

Dionysiac Mysteries 75, 99, 100-09; admission: 102; appas: 106; bath: 104; biennial: 102; boukolos: 109; cista mystica: 104, 108; chorêgos: 106; cistiferi/ kistophoroi: 92, 104-05; dancing: 105-06; date: 103; drinking: 108; fawnskin: 108: goat: 105; grades: 103, 109; grotto: 103; grove: 103; hall: 109; heartland: 100; hierophant: 101, 107; hieros logos: 105; hypotrophos: 106; liknon: 108; liknophoros: 104; lychnaptria: 106; and maenadism: 102; men: 102; music: 106; mystês/ai: 100, 105; narthêkophoros: 104; nocturnal: 105; no oath: 105; orgiophant: 107; without Orphism: 78; phallophoros: 104; phallus: 108; phasmata: 107; play: 106-07; priestesses: 102, 105; procession: 104; pyrphoros: 104; sacrifice: 104-05; sebastophant: 107; secrecy: 103, 108; simiophoros: 104; snake: 108; symbola: 109, 151; theophant: 107; theophoroi: 104; thiasôtês: 100; thyrsophoros: 104; Titans: 105, 108; women: 102, 105

Ps.Dionysius Areop. EH 3 A.B: 162

Dionysius Hal. AR 1.61.4: 33, 2.22.2: 36; De compos. verb. 6.25.5: 63

Dionysodoros FGrH 68 F 1: 35

Dionysos 81; and Attalids: 109; D. Bakchos/ Bakcheus/Bakch(e)ios: 70-72, 75-77, 79, 106, 203; D. Euios: 71; murder of: 76-77, 105, 108-09, 152; and Osiris: 111; parents: 62; Thracian: 56; in underworld: 76; youth: 106 
Dioskouroi piloi: 93

doves and colonisation: 195

Ecphantides, ed. Kassel/Austin fr. 4: 71

Egyptian Mysteries IX, 110-11, 142, 144; and Enlightenment: 144; and Khoiak festival: 110-11; non-existent: 110; Osiris: 110

Egyptian priests 118; books: 119

Elektra 36

Eleusinian Mysteries XII, 1-20; admission: 2-3, 176; and agriculture: 19; allegorisation: 2; anaktoron/a: 8, 12-14, 31, 168; and Augustus: 196; Baubo: 35; birth of child; 12, 14-15; Brimo/os: 14-15, 35, 73; Celeus: 12; chthonios hymenaios: 12 ; clothes: 17 ; coins: 17 ; daidouchos: 124 , 162; dancing: 7, 10, 13; Demeter, statue of: $12,14,107,154-55$; ear of wheat: 14 , 107; end of: 163; ephebes: 6, 8; ерорteia: VIII, 11-16, 67, 162; and eschatology: 19-20; fee: 4; and fertility: 18-20; fire: 14, 123, 151; goal: 18: 18-19; hierophant: 11, 13-15, 163, 167; hymns: 6, 13, 154; and Imperial Mysteries: 154; Kallichoron Well: 7-8; Kronos: 12, 14; megaron/magaron: 12-13, 168; mocking: 7; myêsis: 5-11, 67; myrtle: 6, 16: 16; mystic drama: 10,96 ; mystic utterance: 17 ; night: 7 ; officials: 10 ; Orphic influence: 2, 15, 65; paks/konks: 17; panegyris: 17, 96; participation into: 4; Petra Agelastos: 176; phallus: 11; pig/lets: 5, 17; and Plato: 1-2; Plemochoai: $16-17$; preliminary initiation: 3 ; priests/esses: 6,16 ; procession: $5-6$; proclamation: $4,136,182$; Propylon: 7; purification: 6,196 ; reconstruction: 1-2; sacrifices: 8; sanctuary: 168 ; search of Persephone: 11, 28; secrecy: 17-18, 163; seeing/showing: 77; shuddering: 13-14, 162-63; staff: 6, 17, 196; study of: VIII-IX; symbolon: 3, 121; telestêrion: 9, 15, 26-27, 77; terrifying experience: 13-14; torches: 11; Triptolemos: $12,15,17,19$, 113; two nights: 9-10; and Virgil: 196; wisdom: 18; wreath: 16 elm 183

Elpenor 187

Elysium 196-99, 202-03

embateuô 150

Empedocles, ed. Diels/Kranz B 3.3-5: 77 and 182, 115: 200 and 202, 118-21: 202, 121: 183, 127: 197, 140: 197, 142: 188, 147-48: 203

Eniautos 19

1 Enoch 185, 203; 17-18: 199

Epaminondas 87

Ephorus FGrH 70 F 80: 5, F 120: 23, 28

Epigenes 60, 64

Epiphanius Pan. 26.13.2: 159

Epiteles 87, 96

Eratosthenes Cat. 39

Eridanus 197

Erman, A. 145

Etymologicum Magnum s.v. Magnêtis: 29; Methodios: 176; Nyktelios: 105

Eubouleus 65

Eubulus, ed. Kassel/Austin fr. 146: 172

Eudoxus $F G r H 79 \mathrm{~F} 5$ dub. $=$ fr. (Lasserre) 338 $=$ Agatharchides $\mathrm{FGrH} 284 \mathrm{~F} 3: 97$

Eumenes II 109

Eumolpos 13, 191

Eunapius VS 476: 163

Euneus/ids 66, 78

Euphorion, ed. Lightfoot 11.13: 191; fr. 14: 62, Euripides library: 68; and Orphism: 68, 79; Alc. 357-62: 60; Ba. 125: 49 and 52, 133-34: 102, 141: 71, 471-72: 182, 474: 77; Cycl. 25: 71; Hel. 175: 62, 1013-16: 201, 1562: 8; Her. 613: 15; HF 15: 194; Hipp. 25: 7 and 15, 141-44: 50 and 97, 952-54: 68, 986-87: 68; IA 1499: 194; Ion 1074: 7; Or. 964: 62, 1086-87: 201; Phoen. 684: 62; Suppl. 54: 15, 533-34: 201; fr. (Kannicht) 248: 89: 370.71: 201, 472.9-19: 66, 752g.8-12: 57, 758a.1103-8: 78, 759a.1619-22: 78; 839.10-11, 908b, 971: 201

Eusebius Contra Hieroclem 30.3: 4; DE 1.10.32, 3.4.48, 4.71: 162; De laude Const. Prooem. 4: 63; PE 3.12: 10, 124 Eustathius Od.1.235, 11.236: 189 
family resemblance XIII

Firmicus Maternus De errore 3.1: 161, 5.2: 137, 6.5: 108, 20.1: 131

Frazer, J.G. 153, 171

Freud, S. 160

Gagné, R. 64

Galen De usu partium 12.6: 63

Ganymede 46

Geertz, C. 1

Gellius 16.5.11.6: 183

Geryon 184

Gilgamesh epic 112

Glykon 155

Gnostics and Mysteries: 158-59

gods anonymous: 34 ; epithets: 179 ; great 34

Gold Leaves and D. Bakchios: 74; and Gnostics: 159; and Italy: 73; and oral performance: 74 ; as passport: 73 ; reincarnation: 75, 200, 203; underworld: 187; and women: 69-70

Golden Bough 194-96

Golden Fleece 195

Gordon, R. XI, 128

Gospel of Philip 159

Graf, F. 1, 51, 74, 108, 145, 157-58, 169

guides 166

Hades 76; house of: 188

Gregory of Nazianzus Carmen de se ipso, PG 37.1367: 63; Or. 4.70, 89: 135; 5.8: 39.4: 11-12, 39.5: 135

Hadrian 154

Hanell, K. 166

Harmonia and Samothrace: 28, 36

Harpocation, ed. Keany $\lambda$ 13: 74

Hecataeus of Abdera FGrH 264 F 25: 119

Hecate 50, 97, 200

Hecate, Mysteries XII, 81, 97-97-99; Eleusinian influence: 99; and Korybantes: 97 , 98; nocturnal: 98; and Orpheus: 97; phasmata: 98; priests: 99; sanctuary: 97; thiasos: 99; yearly: 98

Hellanicus F 5a, b Fowler: 58, FGrH 4 F $23=$ F 23 Fowler: 28, 33, F $87=$ F 88 Fowler: 194

Hephaestus pilos: 93
Heracles 23; and Cerberus: 74; descent poem: 180, 185, 187, 190-93, 196, 198 Heraclides Ponticus, ed. Wehrli² fr. 89: 61 Heraclitus, ed. Diels/Kranz 79, 142; B 14: 70-71

Hermes ithyphallic statues: 31

Hermippus, ed. Wehrli2 fr. 31: 172

Herodotus 2.51: 21, 27, 32 and 33, 2.53: 58; 2.59.2: 111, 2.81: 73, 2.81.2: 67 and 89, 2.144.2: 111, 2.156.5: 111, 2.171.1: 110 and 173, 2.171.2: 174, 4.78-80: 72, 4.79.3-4: 72, 7.6.3: 192, 7.153: 167, 8.65.1: 6: 9.80: 190

Hesiod Op. 167-73: 202; Th. 504-05: 194, 726-27: 188, 769-73: 186, 969-71: 33; fr. (Merkelbach/West) 15: 189, 30: 189, 78: 189, 177: 36, 280: 192

Hesychius, ed Latte et al. $\alpha$ 431: 176, 2727: 5, 4390: 13, 5234: 173; ठ 2036: 175, З 145: 174, k 3098: 173, 3134: 17; $\mu$ 1355: 127 , $\rho$ 202, б 1825, 1827: 172

Hesychius of Jerusalem Hom. pasch. 1: 162

Hieronymus, ed. Wehrli2 fr. 42: 61

Hieronymus 134, 137; Adv. Iov. 1.7: 131; Ep. 107.2: 133

Himerius Or. 67.9: 14

Hippias 86 B 6 DK: 58

Hippolytus 7, 68, 80

Hippolytus Ref. 5.7.3: 39, 5.8.9: 27, 5.8.39-41: 9, 14; 5.9.8: 161, 8.22-24: 161

Hipponax fr. $\left(\right.$ West $\left.^{2}\right) 155 b=\left(\right.$ Degani $\left.^{2}\right)$ 164: 35; fr. dub. (Degani2) $197=$ Adesp. lamb. (West $\left.^{2}\right)$ 58: 35, 36

Homer 58; Nekuia: 180, 190; underworld: 184; Il. I.403: 183; VII.131: 188, 467: 40; VIII.15: 188; XI.263: 188; XIV.279: 202, 457: 188; XX.366: 188; Od. 5.125: 33; 7.324: 189, 10.509: 196, 513: 188; 11.37-41: 187, 51: 187, 576: 189; 24.12: 183

Homeric Hymn to Demeter 47: 11, 153: 177, 474: 177, 476: 182, 477: 177, 478-79: VII, 480-83: 19-20, 480: 15 and 18, 489: 15

Horace and Jewish literature: 204; C. 2.14.8: 184, 3.1.1: 63 and 188, 3.4: 189, 3.4.80: 192, 3.8.1-8: 104 
Hornung, E. 113

Horsfall, N. 181, 195

Hose, M. 148

Hyginus Fab. 61: 189, 79: 192, 167 and 179: 123

lakchos 6-7

lamblichus Life of Pythagoras 151: 38, 153 : 89; Myst. 3.9: 52

Idomeneus FGrH $338 \mathrm{~F}$ 2: 14

Imbros, Mysteries 37-38; Great Gods: 37; Hermes: 37-39; Imbrasos: 37-38; Kabeiroi: 37; Lord Kasmeilos: 37; purifications: 38 ; secrecy: $37-38$; Titans: 37

Imperial Mysteries 154-55

Inanna 153

initia VII

initiation and Mysteries: VII-VIII, 77; and wolves: 77

inscriptions Année Epigraphique (AE) 1953.238: 99, 1978.641: 137, 1979.425: 137, 1980.48: 137; Agora 15.78.6-8: 172, 16.239: 16; BCH 4 (1880) 399 no. 8: 50; CIL III.686: 105, CIL VI.261, 504, 507, 510-11 and 1675: 99, 2255: 104, 30966: 98, 31940: 99; CIL X.1583: 107; CIMRM 42: 134, 54: 137, 60: 137, 63(a): 137, 65: 137, 333: 137, 403: 137, 423: 137, 397: 134, 423: 137, 475: 137, 511: 137, 622: 137, 626: 137, 688: 136, 706: 137, 708: 137, 774: 137, 793: 137, 803: 137, 1083: 137, 1098: 128, 1137: 137, 1243: 137, 1292: 137, 1359: 137, 1400: 137, 1430: 137, 1579: 137, 1584: 137, 1718: 128, 1896: 134, 2269: 128; Hansen, Carmina II.535, 545, 558 and 593: 201; I. Anazarbos 9.7-9: 137; I. Ancyra 8: 155; I. Eleusis 19: 2-4, 177: 2, 208: 5, 7 and 8, 233: 4, 250: 5, 300.18: 13, 515.4: 13, 637.1-2: 14, 638.29-30: 6, 659.10: 14; I. Ephesos 1268: 104, 1601-02: 104, I. Erythrae 201: 48 and 50, 204: 52, 206: 48, 50; IG $I^{3} 6$ : 2-4, 84.35-36: 5, 1179.6-7: 201; IG II2 674: 172, 847: 5, 7 and 8, 1078.29-30: 6, 1177.23: 173, 1184: 175, 1672: 2, 3639.4: 13; IG II/III2 3709.10: 14, 3811.1-2: 14; IG
V 1.1390: 87-96, 1467-69: 91; IG V 2.281: 154, 312: 154, 514: 83, 516: 82; IG VII.3596-97: 45, 3599: 46, 3626: 46, 3970: 46, 4122: 45; X.2.1, 103: 114; IG X 2.254: 112; IG X 2.1.260: 105; IG XII 1.6.1197: 48, 50, 53; IG XII 4.1.299: 49, 52; IG XII 5.14: 112; IG XII 6.1197: 50; IG XII 6.2, 600: 114; IG XII 8.74: 35 and 37, 8.51-52: 37, 8.68-74: 37, 8.70: 38, 8.87-89a-b: 37-38; IG XIV 268: 178, 1815: 118; IGASM 39: 178; IGBulg 1.23: 105, 370bis: 177, 401: 105, 2.1517: 104-5 and 107, 3.1517: 105, 4.1862: 106; IGLMP 49: 178; 56: 178IGUR 160: 92, 104-5; I. Klaudiopolis 7, 56 and 65: 154; ILS 1259-60, 1264: 99, 3364: 107, 3368: 105, 4148: 99; I. Magnesia 117: 106; Inschriften von Milet 171-74: 43; I.

Pergamon 485: 106, 486a: 106; I. Prusa 48: 114 and 120; I. Samothrace 29.13-16: 33, 168-69: 30, 170: 23; I. Smyrna 728: 67, 105, 107; IvP 251-52: 41; I. Stratonikeia 541: 107; I. Tralles 86: 114, 180: 118; Jacottet, Choisir Dionysos, 4: 102 and 106; 22: 102 and 105, 29: 105, 31: 103, 45: 102 and 106, 46: 105, 47: 104-5 and 107, 54: 102-3, 58: 102-3, 94: 106, 98: 102, 99: 102 and 106, 108: 104, 112: 106, 126: 105 and 107, 137-9: 104, 147: 102 and 106, 149-50: 102, 152: 103-4, 156: 102, 163: 102, 169: 102, 172: 107, 174: 102, 181: 102, 185: 104, 188: 102-4; LSCGS 15: 3; LSS 15.42: 7; Merkelbach/ Stauber, Steinepigramme I, 06/02/01: 54, II, 10/03/02: 71; OGIS 2.764: 41; RICIS 101/0206: 116, 101/0221: 116, *102/ 0201: 114, 113/0537: 140, 113/0545: 112, 114/0202: 112-3, 202/0139: 120, 202/ 1101: 112, 202/0209: 116, 202/0428: 121, 204/1002: 120, 205/0104: 114, 302/ 0204: 111, 303/1301: 114 and 140, 306/ 0201: 112, 308/0401: 114, 120 and 140, 308/1201: 114 and 122, *312/0501: 114, *501/0127: 114 and 140, 501/0165-66, 501/0168, 501/0185, *501/0188 and *501/0190: 114, 503/0301: 121, 505/ 0101: 114, 512/0201: 114, 512/0602: 114, 
Suppl. I, 113/1201: 111; SEG 6.59.21: 150; 12.411: 178; 28.141: 103-4; 29.795: 22; 30.93.18: 13; 32.1236-37: 118; 36.376: 82; 38.1218: 118, 39.737A: 34; 40.146.247-8: 26; 43.630 A 17: 26; 45.897-98: 34; 45.1194: 38; 46.810: 48 and 50, 1519: 118; 47.1628: 48 and 50; 50.168 A II.44: 26, 831, 836-37: 38; 51.328: 199; 52.1146-47, 1590: 137; 53. 53.48 A.fr.3.III.71: 177, 1786, 27: 62; 56.418: 167; 57.64 B 12: 64, 1164-65: 118; 58.1664: 118; 59.947: 37, 1200: 38; Syll. 3 735.21-22: 95; Tituli Asiae Minoris (TAM) V 1.477: 106, 822: 104; Tit. Cam. 226 no. $90.134: 50$

lobacchants 102,106

Ion of Chios B 2 DK = FGrH 392 F 25a: 73

Isaeus 3.80: 175, 6.50: 173

Isidore Or. 19.32.5: 29

Isis abstention: 120; aretologies: 111-13, 121; in Cenchreae: 115; and dreams: 116; and Eleusis: 113; followers: 140; and Hecate: 121; as inventor: 113; I. Orgia: 114; in Kyme: 112; in Memphis: 112; and Mysteries: 111, 113; and Proserpina: 121; regina manium: 121 ; sanctuaries: 115 , 124; and water: 120

Isis, Mysteries XIII, 110-25; banishing uninitiated: 120; bath: 119; books: 118-19; bricolage: 116 ; clothes: $115,120,124$, 139; dreams: 115-16; Egyptian aspects: 140; elements: 122 ; epicenter: 114 ; fasting: $116-17,120$; fee: $116-17,119$; few and late: 113-14; forgiveness: 120; and gods: 123-25; shaven head: 139; nocturnal: 120; and Sun: 124; sun at midnight: 123; symbolon: 121; torch: 123

Isocrates 4.28: 19, 4.157: 4

Istros FGrH $334 \mathrm{~F}$ 29: 16, F 71: 194

luba $\mathrm{FGrH} 275 \mathrm{~F} 88: 36$

Ixion 189, 193

Jaccottet, A.-F. 101, 109

Jason 23, 57

Jeanmaire, H. 53

Jesus 142-43; and Buddha: 142; resurrection: 161; and 'rising and dying gods': 152-54
John Chrysostom Adv. Iud. 4.7: 162; Cat. Bapt. 2.17 and 3.16, 17: 162; De paenitentia 6: 162; De sanctis martyribus: 162; Ep. ad Olymp. 10.3: 162; Hom. 23 in Eph.: 163; Hom 25 in Eph.: 163

Jong, K.H.E. de VIII

Julian Or. 7.25: 4

Justin Martyr Apol. 1.61.12: 151, 1.62.1: 157, 1.66.4: 157; Dial. 70: 131

Juvenal 6.533: 121

Kabeirides 39

Kabeiro 35, 39

Kabeiroi and Cybele: 36, 38-39; and Dioskouroi: 47; dancing: 48; drinking: 48; etymology: 47; geography: 47; and Hermes: 35, 93; on Imbros: 35; and Hephaestus: 35, 39; and initiation: 54; and Kabeiro: 35, 39; Kabeiros: 48; and Kamillos: 39; and Kasmilos: 47; and Korybantes: 28, 40, 48; and Kouretes: 40, 48, 54; ram: 41-42; and smiths: 48 ; and divine triad: 47 ; two gods: 48

Kabeiroi, Mysteries 21, 37-48; see also Imbros, Lemnos, Miletus, Pergamon and Thebes

Kadmos house of: 167-68; and Samothrace: $23,27,36$

Kaukon 87

Kern, 0. 46

kl(e)ision 77

Korybantes 35; dancing: 49, 52; etymology: 49; fee: 50; geography of: 49 ; iconography: 49; and Kabeiroi: 28; and Kouretes: 49; in Lycosura: 85; and madness: 50; name: 49; nature: 49; weapons: 49

Korybantes, Mysteries XIII, 21, 48-53; ablutions: 50-51; admission: 50; dancing: 52; dining: 52; in Erythrae: 50, 52-53; Euphronisioi: 52; healing: 54; kekorybantismenoi: 53; kratêrismos: 51; music: 52; nocturnal: 52; private: 54 ; sacrifice: 50-51; and Samothrace: 36; Thaleioi: 52; in Thessalonica: 50, 53; thronôsis: 24, 51-52; and women: 53 Korybas 36 
Kouretes dancing: 49; iconography: 49; and Kabeiroi: 54; and Korybantes: 49; in Lycosura: 85; weapons: 49

Kritolaos FGrH 823 F 1: 24, 28

Kronos tower: 188

Lactantius Div. inst. epit. 18.7: 11

Lambrechts, P. 153

Last Supper IX, 146, 152, 157-58; as mystêrion/mysterium: 162-63

laurels 197

Lemnos, Mysteries XII, 38-41; Artemis: 38; Cybele: 38; dancing: 40; Great Gods: 38; Hermes: 39 ; and Hephaestus: 39,47 ; Kabeiroi: 38; kantharoi: 40; Lemnos: 38; Lords Gods: 38; nocturnal: 40; ram: 26; ring: 41; sacrifices: 40; and Titans: 39; Telesterion: 27, 39; wine: 40; and wood: 40 Lesser Mysteries 3

Lethe 199

Libanius 13.19, 52: 4; 14.5: 99

Livy 39.8.4: 105, 39.9: 120, 39.15.13 and 18.3: 105, 45.5.4: 24

Lloyd-Jones, H. 191

Lobeck, C.A. IX-X

Loisy, A. X

Longinus Subl. 39.2

Lucian Alex. 24: 161, 35: 15, 38-39: 9; Cataplus 22: 13, 191; Dem. 11: 4; Dial. Mer. 2.1: 173; Nav. 15: 99; Per. 11: 158; Philops. 13: 161; Pisc. 33: 10; Salt. 14: 10, 15: 105-06; VH 2.29: 192

Lucretius 1.115: 183, 6.1044: 29

Lycophron 162: 35, 36

Lycosura, Mysteries 81-85; admission:

82-83; Anytus: 84; Artemis: $84-85$; box: 85; ecstatic dancing: 85; Demeter: 84-85; Despoina: 82-85; fee: 82; Great Mother: 85; Hall: 83; Hermes: 93; hieros logos: 85; and Korybantes: 85; and Kouretes: 85 ; nocturnal: 85 ; priest/ess: 82 , 85 ; procession: 83 ; sacrifice: $83-84$; Titans: 84-85; women: 83

Lydus Mens. 3.26: 131

Lykomids, Mysteries $13,77,87$; and Methapos: $90-91$; and Orphism: 66,77

Lysias 6.51: 6, 15
MacMullen, R. XI

Macrobius Sat. 3.1.6: 194, 3.20.3: 183

maenads/ism 100-03; in Olbia: 71

Mani 159

Maximus of Tyre Diss. 32.7: 106, 38.2: 52, 39.3: 14

Megara 166-79; and Agamemnon: 176; Anaklêthra: 175-76; and Athens: 177; and Eleusis: 176-77; etymology: 169-70; and Iphigeneia: 176; mythology: 176

Megareus 171

megarizein 168

megaron/magaron 12-13, 168-69, 171

Melanthios FGrH 326 F 4: 17

Meleager 191

Menander Sic. 273: 52; Theoph. 28: 52; fr. (Kassel/Austin) 500: 3, 553: 13

Mesomedes H. 5: 12

Messene 87, 91

Methapos 87, 90, 93

Meyer, E. 144

Michels, A. 195

Miletus, Mysteries 42-43; Assessos: 42-43; Athena: 43; chest: 42; Dioskouroi: 43; Hephaestus: 42; Kabeiroi: 42-43; Tottes and Onnes: $42-43$

Minyas fr., (Davies/Bernabé) 1: 184, 7: 192

Mithras, Egyptian priest 117-18

Mithras, god X, 110; attendants: 129; birth: 131; clothes: 129 ; as creator: 130 ; etymology: 126; Invictus: 140; and Mithrakana festival: 129; and Paul: 143; Persian aspects: 129 ; onomastics: $118,126-27$; origin: 125-26; and polytheism: 139-40; statue: 128 ; in Tarsus: 143

Mithras, Mysteries XIII, 98, 99, 125-38; and astrology: 130, 137; and banquet: 130-31; bread rolls: 158; Bridegroom: 133-35; and caves: $129-31$; and Christianity: 157-58, 164-65; and cosmology: 130; crown: 135; exclusivity: 164; grades: 133-36; grapes: 158; handshake: $136-$ 37; humiliation: 135 ; incense: 136 ; lighting: 135; Lion: 133-37; Mithraea: 129; origins: 128-29; participants: 132; Pater/ Father: 118, 133-38, 163; Perses: 133-37, 140; Persian aspects: 140 ; 
priest: 137; purifications: 136; Raven: 129, 133-35; relief: 128,130 ; sacrifice: 130, 137; Schlangengefäss: 135; and Sol: 130, 134; Soldier: 133-35; Sun-runner: 133-37; tattoo: 139; and women: 131-33; and worshippers: 139-40

Mnaseas fr. 17 (Cappelletto) $=$ FGrH 154 F 27: 35, fr. 41: 36

monstres 183

Mountain Mother 50, 75, 97; and Dionysos: 75

Müller, F.M. 59

munnae VII

Musaeus 58; in Aeneid VI: 191-92, 198-99, 203; books: 69; and Selene: 201; fr. (Bernabé) 52: 192, 68: 192

myêsis VIII, 5-11

myô VII

mystagogue 3-4

Mystêria VII, 21, 70, 150; and objects: 107

Mysterienreligion IX, 164

Mysteries aims: XIII; apparitions: 98, 107; attention to: XIII; books: 119; and baptism: 152; and Christianity: X, 143-65; definition: XI-XII, 138; Egyptian: IX, 110; emotionally impressive: XII; in Ephesus: 154; fee: XII, 138-39; and Gnostics: 15859; and Hermes: 93; and individuality: XII, 138; and initiation: VII-VIII, 77, 102; location: XII; mobility: XII-XIII, 138, 148; and Mousai: 13; nocturnal: XII, 9, 105; open to all: XII; paradidômi: 98, 157; and Philo: 149; and philosophers: 156; and Plato: 148; popularity: 154; and purification: XII; ram: 26, 41, 93; rewards: XII; and sacraments: 163; secrecy: VIII, XII, 152,182 ; standing of: 156 ; survival: 163-64; terminology: 148; and theologians: IX-X; and tribal initiation: VIII; voluntary: XII; see also Alexander of Abonuteichos, Andania, Bacchic Mysteries, Dionysiac Mysteries, Eleusinian Mysteries, Hecate, Imbros, Imperial Mysteries, Isis, Kabeiroi, Korybantes, Lemnos, Lycosura, Lykomids, Miletus, Mithras, Orphic-Bacchic
Mysteries, Pergamon, Samothrace, Thebes

mystêrion cognitive content: 159; etymology:

VII; in Septuagint: 149; in NT: 150, 152

mysterium VII

mystery as detective story: VII

mystery religion $\mathrm{IX}-\mathrm{X}$

mystês 1; as hermit: 163

myth and ritual 19

Myron 97

Nicolaus of Damascus FGrH 90 F 52: 42

Nightengale, F. 59

Nikephoria 41

Nilsson, M.P. 86

Nock, A.D. 147-48, 150-51

Nonnos D. 3.43-51: 27, 3.169-71: 29, 4.184-85: 27 and 40, 4.271: 105, 9.114: 105, 12.391: 105, 13.7: 105, 13.143-45: 49, 13.402: 27, 14.18: 27, 14.19-22: 39, 14.291-92: 105, 16.401-02: 105, 17.195: 39, 19.191: 196, 29.213-14: 27

Norden, E. 59, 61, 180-81, 190-95, 197-201, 204; and Judaism: 204

Obbink, D. 65

Olbia Bacchic Mysteries: 71-72, 79; bone tablets: 55, 71-72; thiasos: 72

Olympias 22-23

Orcus 183, 204

Orient $\mathrm{X}$

Ophites 158

Oracula Sibyllina 3.785: 196, 397-104: 204

Oriental religions $\mathrm{X}-\mathrm{XI}, 164$

orgia VIII; as objects: 75,107

Origen CCels. 3.16: 52, 3.36: 154, 3.59: 4 and 158, 4.10: 14, 6.22: 98, 6.24: 158

Orpheotelests $69-70,74,76,80,87$

Orpheus and Argo: 57; bearded/beardless: 57; of Camarina and Croton: 60; descent poem: 180, 190-92, 196-98; and Euneus: 78; and Eurydice: 55, 57, 60, 79; and initiation: 57; inventor Mysteries: 58, 97; katabasis: 60, 79; musician/singer: 57-58; and Samothrace: 23; son Calliope: 57; as Thracian: 56, 57 
Orphic Argonautica 28-30: 40, 40-41: 61, 466-70: 23, 467: 27

Orphic-Bacchic Mysteries XIII, 70-80, 100; and Aeneid VI: 180-204; convergence East and West: 79; ecstatic dancing: 74; and Egyptian Mysteries: 111; and Gnostics: 159; hearing: 77; murder of Dionysos: 76-77, 105, 111; symbola: 73 , 108, 151; and upper class: 80 ; wreath: 74; see also Bacchic Mysteries

Orphicorum Fragmenta, ed. Bernabé 1(a): 63, 77, 162 and 182, 3: 182, 19: 182, 30: 192, 34: 62, 36: 61: 197, 62, 63: 192, 69: 192, 70: 192, 89: 63, 65: 78, 314-15: 108, 338: 76 and 200, 365: 72, 406: 64, 412: 61, 421: 64, 424: 69, 429-30: 199, 463-65: 55, 463: 71, 465: 71, 467: 76, 474-96: 56, 474.16, 74 and 188: 67, 475: 151 and 187, 476: 74 and 187, 477: 187, 484a: 187, 485-86: 74, 485: 203, 487: 80, 187 and 196-97, 487: 203, 488-91: 75, 488: 76, 80, 200 and 203, 489: 203, 490: 76, 200 and 203, 491: 203, 493: 67, 73, 76, 197 and 200, 493(a): 67, 75 and 151, 496: 67 and 203, 510-23: 58, 528: 67, 531: 77, 567: 66, 573: 74, 576: 67, 577 I: 51 and 68, 578: 151, 589: 63, 627: 68, 650: 73, 654: 70, 655: 74, 666: 69, 707-08: 60, 717: 60, 183-87, 190-93, 198, 200; 800-03: 64, 810: 65, 870: 60, 871: 58, 902-11: 57, 980: 60, 972: 78, 1003: 57, 1018: 64, 1100: 64, 1103-04: 60, 1109: 192, 1128: 60, 1146: 58

Orphic Hymns 6.11: 107, 31.5: 107, 39.3: 52; 44.6-9: 106

Orphikoi $71-72$

Orphism 55, 58-70; and afterlife: 79; and Aither: 78; anthropogony: 76; in Athens: 59-70; bakchos: 66-67, 74-75; books: 68-69; and Eleusis: 2, 15, 65; and Eros: 78; and Euripides: 68; guilt: 76; hieros logos: 73; hymn on Demeter: 65; Hymns: 65; and Italy: 60-62, 65; katabasis: 5961, 187; knowledge: 76; lifestyle: 66-69, 71, 79-80; and light: 196-97; linen: 67-68; mystai/ês: 67, 74-76; narthêkophoroi: 66; and Night: 78; and
Parmenides: 62, 78; Physica: 64-65; and Plato: 66; purity: 67-68; and Pythagoras/eanism: 73, 79; and reincarnation: 72, 76, 80, 199-203; term: 59; Theogony: 61-64; thiasos: 75; and Titans: 76-77; underworld: 196-97; vegetarianism: 67-68; and Virgil: 180-204; and women: 69-70, 80, and youth: 68-69

Orphismus 58-59

Osiris 152

Otto, R. 144

Ovid $A A$ 2.601-04: 27; Am. 1.8.74, 2.19.42, 3.9.33-34: 120; F. 1.353: 104, 1.394: 102, 1.629: 89, 5.441: 75; Met. 1.747: 121, 3.256-315: 123, 5.538-550

Pallas 131-32, 134, 136

Pan 50, 97

Panyassis fr (Davies) $9=$ (Bernabé) 14: 192 papyri P. Col. Zen. 19.2: 175; P. Mich. Inv. 1447.42-3: 191, P.Oxy. 10.1241: 194, 11.1380.110-11: 113, 15.1802.64: 127

Parker, R. 1, 166, 168, 172-73, 175, 191

Pattison, M. 145

Paul and Mysteries: 150

Paulina 99

Pausanias and Mysteries: $81 ; 1.4 .6: 41$, 1.22.7, 31: 77, 1.38.6: 8, 1.39.5: 166, 168 and 171, 1.41.2: 166, 1.43.1: 176, 1.43.3: 176, 1.44.2: 167, 1.44.3: 177, 2.20.3: 166, 2.25.8: 194, 2.30.2: 97, 4.1.5-9: 77 and 87, 4.1.6: 91, 4.1.8: 93, 4.3.10: 95, 4.16.2: $90,4.26 .6-8: 88,4.27 .6: 88$, 4.32.6: 89, 4.33.4-5: 86,90 and 93, 5.27.5-6: 127, 8.9.7-8: 154, 8.31.6: 93 , 8.37.4-5: 84, 8.37.8-9: 82, 8.38.12: 84, 9.16.5: $167,9.19 .5: 178,9.25 .5: 43$ and 46, 9.25.6: 46, 9.25.8: 93, 9.27.2: 77, 9.30.12: 77, 10.15.2-3: 109, 10.28.2: 192, 10.32.9: 116, 10.38.7: 38

PCG, Adesp., ed. Kassel/Austin 1063.15-16: 29, 1146.21-22: 29

\section{Peloponnesus, Mysteries XII}

Peregrinus 158

Pergamon, Mysteries 41-42; and Dionysiac Mysteries: 109; ephebes: 41-42; Kabeiroi: 41; Kabiria: 41; Kriobolia: 41; Meter 
Basileia: 42; myêsis: 41; ram: 41-42; sacrifice: 42

Persephone/Proserpina 10, 62; rape of: 62-63; search for: 11 ; spelling name: 62 , 169; in Aeneid VI: 194, 196

Pettazzoni, R. VIII

Phaedra 7, 50, 97

Pherecydes FGrH 3 F $48=$ F 48 Fowler: 28, 39, 49, F 55 Fowler: 189, F 167 Fowler: 58 Philip II 22-23

Philo and Mysteries: 149-50; Cher. 42: 63; De vita cont. 3-4: 52; Somn. 1.191: 182; SpecLeg 3.2: 199

Philochorus FGrH 328 F 18: 192, F 77: 65, F 185: 65; F 208: 201

Philodemus Piet., ed. Obbink 4967: 72, col. 248 III.13-6: 176; On Poems 1.181: 74

Philostratus VA 4.18: 5, 4.45: 161, 8.7.6: 89; VS 103: 15, 587: 14, 601: 13

Phlegyas 193

Phoroneus 166-67

phôtisma/os 151

Photius, ed. Theodoridis $\theta$ 134: 172 , к 3: 39; K 1063: 51, $\lambda$ 216: 74, 5: 13, $\mu$ 100: 74

phrikê/phrikôdês/phriktos 13-14, 162-63

Phrygian Mother 53

Phryne 5

Phylarchos FGrH 81 F 69: 51

Pindar 0. 2: 61, 21-24: 193, 57-60: 202, 61-67: 202, 68-80: 202, 70: 188, 2.83-85: 182; fr. (Maehler) 129-31a: 202, 129: 197, 133: 76, 137: 15, 19; 196a.7: 194, 266: 194, 346: 191

Pirithous 192

Plato 148; and moon: 201; Ap. 26d: 68; [Axioch.] 365e: 199; Crat. 400c: 199; Crito 54d: 52; Ep. 7, 333e: 3; Euthd. 277de: 50, 148; Grg. 493b: 19, 493ac: 61, 524a: 187 and 197, 525c: 193; Ion 533e: 52, 536c: 52; Leg. 7.790d: 50, 53; Meno 76e: 148, 81a: 69; Phd. 62b: 199, 67d: 199, 69c: 67, 81be: 199, 82b10-c1: 203, 92a: 199, 108a: 187, 113d-114c: 203, 114a: 193; Phaedr.: 9, 247a7: 13, 248-49: 203, 248ab: 16, 249a: 200, 250b6: 13, 251a: 13, 252c3: 13, 254b: 14; Prot. 316d: 192; Resp. 364e: 58 and 201, 364b-e: 69, 10.614bff: 203, 10.614cd: 187 and 203, 10.615b: 199-200, 10.615c: 198 , 10.616a: 193 and 203, 10.616b: 197 and 199, 10.621a: 200; Symp. 215cd: 52, 218b: 63, 77 and 182; Tht. 155ef: 148; Tim. 41e: 199

Pliny $N H$ 11.147: 52, 33.23: 29

Ploutos 15, 19

Plutarch Ages. 24.7: 13; Alex. 2.2: 23, 30.4: 126; Alc. 22: 6, 34.4: 6; Arist. 5: 6; De Fluv. 223.4: 131; Demetr. 26.2; Demosth. 30: 174; Dio 54, 56: 3; Iside 28: 12; Luc. 13.2: 23 and 30, 37.5: 190; Mor. 10f: 9 , 81de: 9, 217d: 24, 229d: 24, 236d: 24, 352cd: 121, 352f: 117, 353d-f: 117, 356b: 111, 364d-365a: 111, 378e: 173, 565e: 103, 566c: 61, 611d: 109 and 151, 635a: 17, 759b: 52, 765a: 3, 780f: 189, 795e: 3, 842a: 7, 943c: 9, 1013f: 7; Phoc. 28: 5, 6, 13; Pomp. 24.5: 127; Them. 1: 77, 15.1: 6; Thes. 10: 177; fr. (Sandbach) 178: 9, 13, 16 and 197, 211: 197

Pluto 19

Poland, F. 100

Polemo De physiognomia, ed. Förster pp.160-4: 161

polis religion VIII

Pollux 1.30-31: 178, 8.90: 4, 8.123-24: 4

Polyaenus 3.11.2: 5

Porphyry Abst. 2.58: 131, 4.6-8: 117 and 125, 4.16: 133; De antro 6: 130, 15: 136; fr. (Smith) 382: 187

Posidippus, ed. Austin/Bastianini Ep. 43.2:14 Posidippus, ed. Kassel/Austin fr. 28: 50, 52 Posidonius, ed. Theiler fr. 368: 3

Priene 53

Ps. Probus, ed. Hagen p. 333-34: 200

Proclus In Plat. Rempl. I, p. 85, 9-10: 109; In Tim. 3.176.28: 17; Theol. Plat. 3.18: 13

Prodicus 65

Propertius 2.33A.1-2, 2.28.62: 120

Ptolemy IV 100

Pythagoraeans clothes: 67,89 ; underworld: 187

Pythagoras descent: 61; dress: 67; and Orphism: 73; and reincarnation: 72 
Radermacher, L. 185

Reitzenstein, R. X

Religionsgeschichtliche Schule 143

Rhea-Demeter 62

sacred law 30-31

sacrifice pregnant victims: 93

Salmoneus 189-90

Samothrace Dionysia: 31; theatre: 31

Samothrace, Mysteries XII, 21-36; admission: 22; and Aeneas legend: 34; Altar Court: 25; Anaktoron: 26, 30-32; Aôoi theoi: 34; asylum: 31; Axieros: 35-36; Axiokersa/os: 35-36; banquet: 33; bronze statues: 27; Daktyloi: 34; dances: 28; dates: 23; Demeter: 33, 35-36; and Diagoras: 28; dining: 29; and Dioskouroi: 23, 29, 35; epopteia: 30-31; fillet: $28-$ 29; fire: 33, 123; gods: 33-36; Great Gods: 35; Hades: 35-36; Hall of Choral Dancers: 25-26; Hermes: 32-33, 35; Hieron: 25-26, 30; lasion: 33, 36; and Kabeiroi: 32-34; Kadmilos/Kamillos/ Kasm(e)ilos: 33, 35-36; kantharoi: 40; Korybantes: 34; Kouretes: 34; lamps: 27, 29; language: 25 ; libations: 26,29 ; lustration: 31; mystai: 30; mythological heroes: 23; night: 27 ; and Odysseus: 28 ; panegyris: 30; Pelasgians: 31-32; Penates: 34; Persephone: 32, 35-36; Perseus: 24; piety: 33; priests: 24 ; procession: 23-24; proclamation: 24; Propoloi: 34; Propylon: 24; purification: 25; ram: 26; ring: 29-30; Rotunda of Arsinoe: 26; Sacred Way: 24; sacrifice: $25-$ 26, 31; and sailors: 28-29; Samothrakiastai: 34; scabrous character: 31-32; search: 28; secrecy: 27; Telchines: 34 ; Telesterion: 31; Theatral Circle: 24, 25, 29; votives, 23, 28, 30; women: 23

Sarapis 12,122

Scaliger, J.J. 47

Scarpi, P. 101

scholia/on Ael. Arist. 3.50: 63, p. 53.15-16: 12; Aesch. 3.130: 5; Ap. Rhod. 1.915-18: 23, 28, 29, 35; Ar. Eq. 408: 6, 785a,c: 176, Plut. 1014: 7, Ra. 159: 6, 330: 16,
369: 4, Thesm. 834: 172, Ve. 1361: 13;

Eur. Alc. 1, Or. 965: 194; Hes. Th. 937 p.

117.7 Di Gregorio: 28; Luc. Dial. Mer. 2.1; Lyc. 211: 106; Pind. O. 7.157: 177, 13.74g: 39, 13.156a,g: 177; P. 3.96: 72, 8.112: 177, 9.161: 177; Soph. Ant. 1146-52: 7, OC 681: 16,173

Scopas 22

Scyles 72

Scymnus 684-85: 33

Selene 201

Semele 106, 123

Seneca HO 597: 102, 1772: 183; Tro. 178: 183: Thy. 909: 190

Servius Aen. 1.297: 32, 1.430: 173; 2.324: 24; 3.12: $27 ; 4.462-63 ; 5.735: 200 ; 6.136: 6$ and 196, 585: 189, 618: 193, 887: 200

SHA Alex. Sev. 18.2: 4; Aurel. 24.3.8: 161; Comm. 9.6: 136; Marc. Aur. 27.1: 4

Sibyl 64; in Aeneid VI: 182-204; as mystagogue: 183

Simonides fr. (Page) 627: 77

Skirophoria 169

Smith, J.Z. 147, 152-53

Socrates 4, 51, 52

Socrates HE 5.19.9: 162

Sopater Rhet. Gr. VII.115.11, 30: 10

Sophists 68-69; and Bologna papyrus: 198; and Mysteries: $148-49$

Sophocles death: 174; Ant. 894: 62, 1134-35: 71, 1146-1152: 7; OC 1049-1051: 7; OT 211: 71; Tr. 219: 71; fr. (Radt) 227: 194, 537-541a: 189, 753.2: 15, 837: 19, 862: 49,52

Sophron, ed. Kassel/Austin fr. 3-*9: 98

Sosibius FGrH 595 F 4: 94

Statius Ach. 1.595: 102, 830: 28; Theb. 1.719-20: 127-28, 5.342-45: 57, 6.706: 193, 12.811: 128

Steiner, R. 142-43

Stenia 172

Stephanus of Byzantium, ed. Billerbeck et al. $\alpha$ 229: 172, 1 57: 35, 37 and 49; s.v. Lêmnos: 38, Nymphai Kabeirides: 39

Stesimbrotus FGrH 107 F 20: 21, 33

Strabo, ed. Radt 7, fr. 20b: 33; 8.6.8: 194, 9.5.21: 193, 10.3.9: 18, 10.3.10: 106, 
10.3.19: 49, 10.3.20: 32, 10.3.21: 37 and

39, 14.1.3: 167, 15.3.13: 127, 15.3.15: 127

Strauss, D.F. 143

Stroumsa, G. 150

Suda, ed. Adler o 49: 176, т 19: 7, X 43: 175

Suetonius Aug. 93, Nero 34.4: 4

symbolon/um 73, 109, 121, 151; and Creeds: 151

syncretism 117

Synesius Aeg. 124b: 105

Tacitus Ann. 3.60-64: 127

Tammuz 152-53

Tartarus 187-89

Tatian Or. 10.1: 63, 27: 10

tattooing 139

taurobolium 161

teletê VIII, 81

Teles 24: 170

Terence Phorm. 48-50: 120

Tertullian Adv. Valent. 1: 11; Apol. 7.6: 27,

8.7: 137; Cor. 15.3: 135

Testament of Abraham 199

Testament of Orpheus 186

Thebes, Mysteries 43-47; admission: 44;

Aetnaeus: 46-47; bulls: 44-45; Demeter Kabiria: 46; drinking: 44-45; fee: 44; and Hephaestus: 47; and initiation: 45-46; and Kabeiroi: 43, 46; Kabirion: 44; Kabiros: 45-46; Kore: 46; and Methapos: 87; Mother: 46; Lemnian influence: 47; nocturnal: 45; and paederasty: 45; Pais: 46; Pelarge: 93; piloi: 93; Pratolaos: 46; priests: 45; Prometheus: 46; purification: 44; sacrifice: 44-45; sash: 45; and smiths: 47; vases: 43-45; wine pourer: 46; wood: 46

Theo Smyrnaeus, ed. Hiller De utilitate mathematicae p. 14.23-24: 4, 15.1-4: 16

Theocritus 4.25c: 172, 7.144: 178, 12.27-33e: 177

Theodoretus Graecarum affectionum curatio 7.11: 11; Hist. rel. 26.13: 162

Theognis 1216: 199

Theophilos, ed. Kassel/Austin fr. 1: 2

Theophrastus Char. 16: 70, 25.2: 29; HP 3.5.2: 183
Theron 61

Theseus 191-93

Thesmophoria 5, 65, 170-77; aition: 168-69, 173; Anodos: 173; and Baubo: 174;

Chalcidic pursuit: 175; and fertility: 175; indecency: 174; Kalligeneia: 174-75; no males: 175; Nesteia: 173; and Orphism: 169; participation: $172-73$; piglets: 174 ; pits: $13,168,171,174$; procession: 173 ; sacrifice: 174-75; secrecy: 174 ; and sexuality: 173; on Sicily: 172; wine: 175

Thomas Magister Ecl. $\varphi$ 378: 62

Tibullus 1.3.26: 120

Timaeus Soph. $\varphi$ 1006b.37-38: 62

Timotheus 12

Tisiphone 191-92

Titans 37, 39, 63; and Orphism: 76; in Tartarus: 197; in Virgil: 188-89, 197; see Imbros, Mysteries; Lemnos, Mysteries, Lycosura, Mysteries

Tityos 189

TrGF Adesp. fr. (Kannicht/Snell) 372199

Tritopatores 64-65

Turcan, R. 101

Turner, V. 7

Valerius Flaccus 1.784: 183, 2.432-33: 27, 2.437-38: 23, 2.439-40: 27

Varro on Samothrace: 27; LL 5.58: 27, 7.34: 36; Rust. 2 praef. 6: 27; fr. (Cardauns) 21: 156, 206: 24, 271: 18

Vermaseren, M.J. XI

Versluys, M.J. 113

Versnel, H.S. 171

Virgil abortion/infanticide: 186; and Apollonius Rhodius: 195; and Bologna papyrus: 190, 198; demonstrative pronouns: 185; doves: 195; and Elysium: 203; and 1 Enoch: 185; and Er: 200, 203; and Hesiod: 188; and Jewish literature: 18586, 199, 204; and Meleager: 195; and Orphism: 180-204; and Pindar: 188; and Plato: 195; and reincarnation: 199-202; sinners in Aeneid VI: 190; and underworld: 184; Aen. 2.460: 188; 4.58: 171, 302: 102 , 303: 105; 6.120: 61, 142-43: 194, 204-08: 195, 208-09: 195, 248-416: 
182-85, 258: 64, 120 and 182-83, 266: 182, 268: 183, 318-20: 185, 322-30: 185, 333-83: 185, 384-416: 185 , 417-547: 185, 417-25: 186, 426-29: 186, 430: 187, 434-36: 187, 438: 184, 447: 187, 450-76: 187, 477-547: 187, 540-43: 187, 548-627: 187-93, 552: 188, 554: 188, 560-61: 185, 563-67: 185, 566: 191, 571: 191, 574-77: 185, 575: 188, 580: 185 and 197, 582: 185, 583: 185 , 587: 185, 595: 189, 602-06: 190, 608-13: 190, 608: 185 and 198, 609: 198, 610: 185, 612-13: 198, 614-17: 190 and 192, 618-24: 190, 618: 193, 621: 185, 623: 185, 628-36: 193-96, 630-31: 193, 635-36: 194, 637-78: 196-99, 639-41: 196, 642-45: 197, 657-59:

196-97, 663: 197-98, 669-70: 191, 678: 198, 679-83: 199, 703-04: 199, 705: 199, 710ff: 185, 724-33: 199, 734: 199,
738: 199, 739-43: 200, 744: 196, 746: 199, 748: 76, 773-74: 185, 776: 185, 697-887: 199-201, 679-80: 199, 788-91: 185, 808: 185, 863: 185, 887: 200; 7.570: 183; 7.323-40: 183; 8.193, 241, 251: 183, 447: 194; G. 2.393: 104; 4.521: 105, 467-69: 61

Weber, M. 80

West, M.L. 60

Wilamowitz 58, 86

women and sacrifice: 175

Xenophon Hell. 5.2.29: 172

Yates, F. 145

Zenobius 4.22: 29

Zeus birth: 54; in Derveni papyrus: 62; Idaean: 66; rape: $62-63,152$ 